\title{
Grain Boundary Influence during Short Fatigue Crack Growth Using a Discrete Dislocation Technique
}

\author{
P. Hansson ${ }^{1, a}$ and S. Melin ${ }^{1, b}$ \\ ${ }^{1}$ Division of Mechanics, Lund University, Lund, Sweden \\ a per.hansson@mek.lth.se, ${ }^{\mathrm{b}}$ solveig.melin@mek.lth.se
}

We have studied the effect of a grain boundary in front of a short edge crack on its propagation under cyclic loading conditions in bcc iron. The used model is a combination of a discrete dislocation formulation and a boundary element approach where the boundary is described by dislocation dipole elements, while the local plasticity is modeled by discrete dislocations. The grain boundary is considered impenetrable, but dislocations positioned in the vicinity of a grain boundary give raise to high stresses in neighboring grains which, eventually, results in nucleation of dislocations and a spread of the plastic zone into the next grain.

Keywords: fatigue, grain boundary, discrete dislocation.

Introduction. It is well known that the behavior of short cracks differs from that of long cracks due to the relative large plastic zone and strong influence of the surrounding microstructure. Experimental studies have shown that short cracks grow through a single shear mechanism [1] and that they can grow at load levels well below the threshold value for long cracks at high rates. They can therefore not be treated by the standard methods used for long cracks.

For very low growth rates, in the order of a few Burgers vectors per cycle only, it is important to account for the discrete dislocations within the material. Authors [2, 3] have developed such a discrete dislocation model for a long Mode I crack to study the cyclic crack-tip plasticity and plastically induced crack closure. A similar model has also been developed in [4] to study the influence of grain boundaries on short mode I cracks.

In this study, a discrete dislocation model, were both the geometry and the plasticity are described with discrete dislocations, is used to study a short edge crack subjected to fatigue loading. The plasticity is in this study restricted to two grains, and the change in growth behavior due to the spread of plasticity between grains is investigated.

Statement of the Problem. The growth of a microstructurally short edge crack located within one grain, subjected to fatigue loading (Fig. 1), has been investigated under plane strain and quasi-static conditions. The crack is assumed to grow in a pure shear mechanism due to nucleation, glide and annihilation of discrete dislocations along slip planes in the material. The initial crack of length $a_{0}$ and inclined at angle $a$ to the free edge normal is located within a semi-infinite body. The load is applied parallel to the free edge and is varied between a maximum value, $\sigma_{y y \max }^{\infty}$, and a minimum value, $\sigma_{y y \text { min }}^{\infty}$. In this study, two neighboring grains are considered, with both grain boundaries parallel to the free edge. The grain boundaries are considered to be dislocation barriers, which the dislocations can not pass and will not contribute to the overall stress field. Nucleation and glide of dislocations is restricted to one slip direction in each grain, inclined at angles $\alpha$ and $\theta$ to the free edge normal and with the slip direction in the first grain coinciding with the initial crack direction.

Initial Conditions. The material in this study is pure iron and is assumed to be linear elastic. The material parameters at room temperature are shown in Table 1 [5] together with the geometrical data for the initial edge crack given in Fig. 1. 
Material Data for bcc-Iron and Geometrical Data for the Edge Crack

\begin{tabular}{|l|c|l|c|}
\hline Shear modulus $\mu, \mathrm{GPa}$ & 80 & Burgers vector $b, \mathrm{~nm}$ & 0.25 \\
\hline Poisson's ratio $v$ & 0.3 & Lattice resistance $\tau_{c r}, \mathrm{MPa}$ & 40 \\
\hline
\end{tabular}

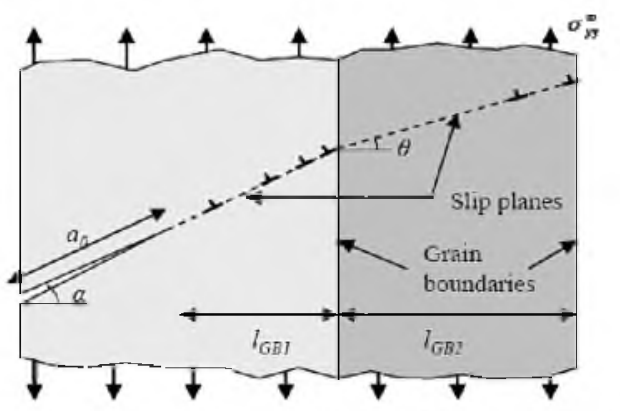

\begin{tabular}{|c|c|}
\hline Initial crack length, $a_{0}$ & $10000 b$ \\
\hline Crack angle, $\alpha$ & $45^{\circ}$ \\
\hline Slip plane angle in grain $2, \theta$ & $30^{\circ}, 45^{\circ}, 60^{\circ}$ \\
\hline Distance to grain boundary, $l_{G B I}$ & $10000 b$ \\
\hline$\sigma^{x} \quad \tau^{\infty} l_{G B 2}$ & $5000 b$ \\
\hline Load range, $\sigma_{y \max }^{x}-\sigma_{y \min }^{\infty}$ & $240-40 \mathrm{MPa}$ \\
\hline
\end{tabular}

Fig. 1, Initial geometry of the short edge crack.

Discrete Dislocation Technique. The model used in this study rests solely on a discrete dislocation formulation, describing both the geometry and the plasticity in the grains by discrete dislocations. The external boundary, defined as the free edge together with the crack itself, is modeled using dislocation dipole elements [6]. A dipole element consists of two glide dislocations and two climb dislocations, with equal size but opposite direction of the two dislocations of same kind. The dislocations are located at the end points of the element, while the stresses in the element are calculated at the element center point.

The stress at an arbitrary point is calculated as the sum of the stress contributions from the physical dislocations describing the plasticity, the dislocations forming the dipole elements and the applied external load. The magnitudes of the dipole dislocations are determined from an equilibrium equation, Eq. (1), describing the normal and shear stress along the external boundary. Knowing that the normal and shear stresses must equal zero along the free edge and along the parts of the crack that is open, the magnitudes of the dipole dislocations can be calculated.

$$
G b_{\text {boundary }}+b G_{\text {internal }}+\sigma=0
$$

In Eq. (1), $G$ is matrix containing influence functions [7], describing the stress field from a dislocation along the external boundary, $b_{\text {boundary }}$ is a vector holding the magnitudes of the dislocations in the dipole elements, $b$ is the Burgers vector of the material, $G_{\text {internal }}$ is a vector containing the influence functions for the physical dislocations, and $\sigma$ is a vector containing the contribution from the applied external load along the external boundary.

Dislocation Nucleation. Nucleation of new dislocations is assumed to occur if the resolved shear stress at a possible nucleation site exceeds the nucleation stress. Dislocations nucleate in pairs, consisting of two dislocations of equal size but opposite sign separated by a small distance $r_{n u c}$. The definition of a positive and negative dislocations nucleated at the crack tip is shown in Fig. 2a. It is assumed that nucleation is possible both in front of the crack tip and at the grain boundary between the two grains.

The nucleation stress is here defined as the lowest stress at the nucleation point for which the positive dislocation in the newly nucleated dislocation pair travels inwards in the material immediately after nucleation. This definition results in a geometry dependence 
of the nucleation stress and, therefore, the nucleation stress is not the same in front of the crack as at the grain boundary. A more thorough discussion on the choice of nucleation stress is found in [6]. In order to determine when, and on which slip plane the next nucleation of a dislocation pair will take place, the resolved shear stress is calculated at all possible nucleation sites for each load level.
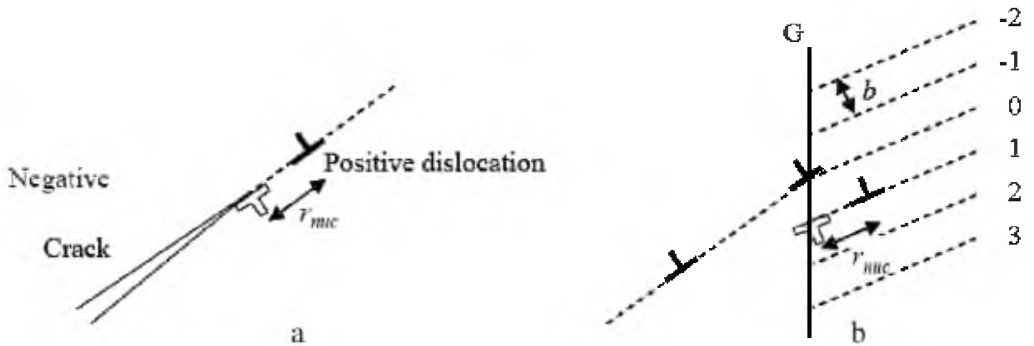

Fig. 2. Nucleation condition and definition of positive and negative dislocation at the crack tip (a) and at the grain boundary (b).

Crack Growth. It is assumed that no dislocations exist within the material prior to the first load cycle. When the applied load gets sufficiently high, dislocation pairs will nucleate from the crack tip. A positive dislocation glides inwards in the material immediately after nucleation along its slip plane as long as the resolved shear stress at its position exceeds $\tau_{c r}$, whereas the negative dislocations will remain at the crack tip. These dislocations shield the crack tip and the load must therefore be increased before more dislocations will nucleate. The positive dislocations pile up at the first grain boundary, resulting in high stresses on the opposite side of the grain boundary. Eventually, these high stresses will result in nucleation of dislocations in the second grain. Also in this grain the positive dislocation will move inwards in the material immediately after nucleation whereas the negative one remains at the grain boundary. This process of dislocation nucleation in the two grains continues until the maximum load is reached and the load starts to decrease. Load reversal eventually results in dislocation glide in the opposite direction, back towards the crack. When a positive dislocation gets sufficiently close to its negative counterpart, the two dislocations annihilate resulting in crack growth in the corresponding direction by one $b$, under the assumption that no healing of the crack surfaces is allowed. A more detailed description of the crack growth model used can be found in [6].

Results. In order to investigate the influence of plasticity spread on the crack growth behavior, the angle $\theta$ shown Fig. 1 is varied to regulate the resistance to dislocation nucleation in the second grain, and the results were compared to results obtained by restricting the plasticity to one grain only. The results of the simulations are presented in Fig. 3, where Fig. 3 a shows the number of dislocations, $N$, in the two grains for different applied load levels during the loading phase, and Fig. $3 \mathrm{~b}$ - during unloading. It can be seen that when allowing the plasticity to spread into the next grain, the number of nucleated dislocations in the first grain increases, as compared to the one-grain model. It can also be seen that a higher number of dislocations are present at the minimum load. This happens because the negative dislocations in the second grain reduce the stress field from the piled-up dislocations in the first grain, allowing more dislocations to exist in this grain. A result of this is, somewhat surprisingly, that the obtained crack growth rate is the same in all four simulations. It can also be seen that at different $\theta$, different numbers of dislocations are nucleated in the second grain. It was also found that no annihilation of dislocations occurred in the second grain during unloading in any case studied and, therefore, the second grain was not included in Fig. $3 \mathrm{~b}$. 


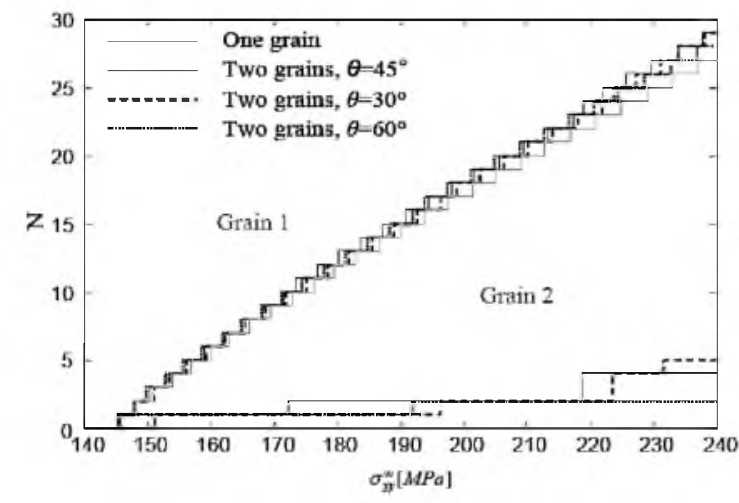

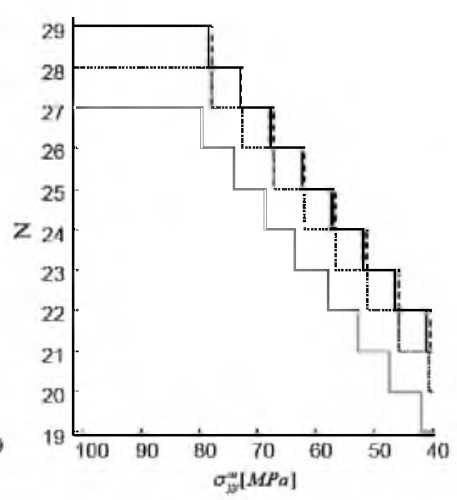

b

Fig. 3. Number of dislocations $N$ as a function of applied external load $\sigma_{y y}^{\infty}$ during loading (a) and unloading (b).

The order in which the slip planes in the second grain were activated, according to Fig. 2b, was also studied. This result is obtained for $\theta=45^{\circ}$, and it was found that the first dislocation for this case nucleated in plane 1 , which is the slip plane just below that in the second grain, coinciding with the slip plane in the first grain (Fig. 2b). The following order of planes on which nucleation occurred was plane 0 , plane, -1 , and plane 2 . As seen, nucleation in the second grain first occurs at the slip planes closest to the slip plane in the first grain before occurring in more distant planes.

Conclusions. The discrete dislocation formulation has been used to investigate the growth behavior of a short propagating edge crack under fatigue loading conditions When investigating the effect of the grain boundary it was found that more dislocations were nucleated at maximum load in the first grain, holding the crack, when allowing the plasticity to spread into the next grain, as compared to when restricting the plasticity to the first grain only. However, the number of dislocations in the first grain also increased when allowing the spread of the plasticity, resulting in the same crack growth rate for the simulated cases. It was also found that the dislocations in the second grain first nucleated on slip planes close to the slip plane in the first grain before continuing in more distant slip planes.

1. D. S. Suresh, Fatigue of Materials, Second Edition, University Press, Cambridge (1998).

2. F. O. Riemelmoser, R. Pippan, and O. Kolednik, "Cyclic crack growth in elastic plastic solids a description in terms of dislocation theory," Comput. Mech., 20, 139-144 (1997).

3. F. O. Riemelmoser and R. Pippan, "Mechanical reasons for plasticity-induced crack closure under plane strain conditions," Fatigue Fract. Eng. Mater. Struct., 21, 1425-1433 (1998).

4. C. Bjerken and S. Melin, "A study of the influence of grain boundaries on short crack growth during varying load using a dislocation technique," Eng. Fract. Mech., 71 (15), 2215-2227 (2004).

5. D. R. Askeland, The Science and Engineering of Materials, Third Edition, Stanley Thornes (Publishers) Ltd (1998).

6. P. Hansson and S. Melin, "Dislocation-based modeling of the growth of a microstructurally short crack by single shear due to fatigue loading," Int. J. Fatigue, 27, 347-356 (2005).

7. D. A. Hills, P. A. Kelly, D. N. Dai, and A. M. Korsunsky, Solution of Crack Problems: The Distributed Dislocation Technique, Kluwer Academic Publisher (1996).

Received 28. 06. 2007 